\title{
Visualising Australia's older population using grid maps
}

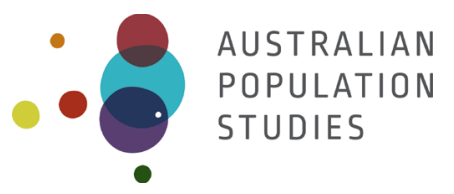

\author{
Anthony Kimpton* The University of Queensland \\ * Email: a.kimpton@uq.edu.au. Address: Queensland Centre for Population Research, School \\ of Earth and Environmental Sciences, Chamberlain Building, The University of Queensland, St \\ Lucia, Qld 4072, Australia. \\ Paper received 17 April 2020; accepted 28 April 2020; published 25 May 2020
}

\section{Introduction}

In 2016, 15.7 percent of Australians were aged 65 or over ( 3.7 million out of a total population of 23.4 million; Australian Bureau of Statistics 2016). This national age structure is already without precedent while projections suggest this could reach 22 percent by 2057 ( 8.8 million; Australian Institute of Health and Welfare 2018). As such, these projections suggest that it will become increasingly challenging for all tiers of Australian government to fund the infrastructure and support services critical for the health and wellbeing of older Australians as they grow at a faster rate than the working age population (Parliamentary Budget Office 2019). There are four critical dimensions when examining population age structures, namely: (1) numbers; (2) characteristics and values; (3) proportions of the population in particular age groups; and (4) spatial distribution (Hugo 2003). In this DemoGraphic I focus on the latter two dimensions to identify where Australians aged 65 and over are most spatially concentrated.

\section{Data and methods}

I draw on the Australian Bureau of Statistics (ABS) 2016 Census of Population and Housing data in Statistical Area Level Two (SA2) neighbourhood units released as open data from their DataPacks web page (ABS 2016). The ABS designed SA2s to approximate functional communities that are connected through regular social and economic interaction, although Australian population density is low within the interior but relatively high towards the coast, with SA2s ranging in area between 49 hectares and 51 million hectares.

Given this variability in area, it has remained a challenge to visualise population structures across Australia. I have therefore generated a gridded population that is typically featured in dasymetric population maps (Silva et al. 2013; Li et al. 2016). Specifically, I converted the population into points located at random locations across all SA2 polygons, aggregated these points to a decimal degreespaced grid, calculated the gridded proportion of Australians aged 65 or over, and then plotted these values using proportional circles. These gridded proportion circles are useful because they reveal the geographic spread of national age structure and normalise the area containing populations to a grid, thus ensuring that there are no overlapping symbols or polygons that are too small to read. In addition, I have introduced marginal histograms (Lambrechts 2019) to this map to reveal the multi- 
modal distribution of the Australian age structure along lines of latitude and longitude, thus enabling a unique spatial examination of Australia's older population.

\section{Key features}

The DemoGraphic (Figure 1) depicts clear spatial patterning. For instance, the symbols scaled according to population structure reveal that there are areas without symbols, indicating that fewer than 1 percent of the gridded population are aged 65 or older. Symbols larger than the 30 percent symbol featured within the legend reveal that there are ageing enclaves throughout Australia where the population aged 65 or older is more than twice the national average of 15 percent, such as the 32 percent circle representing approximately 25,000 Australians residing along the Fleurieu Peninsula, which is located south of the city of Adelaide. This spatial pattern highlights the regional dimension of ageing and echo other work that has outlined this pressing issue for regional communities (Houghton \& Vonthethoff 2017). Lastly, the marginal histograms that reveal the proportion of the population that is aged 65 or older for a given latitude or longitude reveals that these concentrations can range from 5 to 23 percent across the surface of Australia.

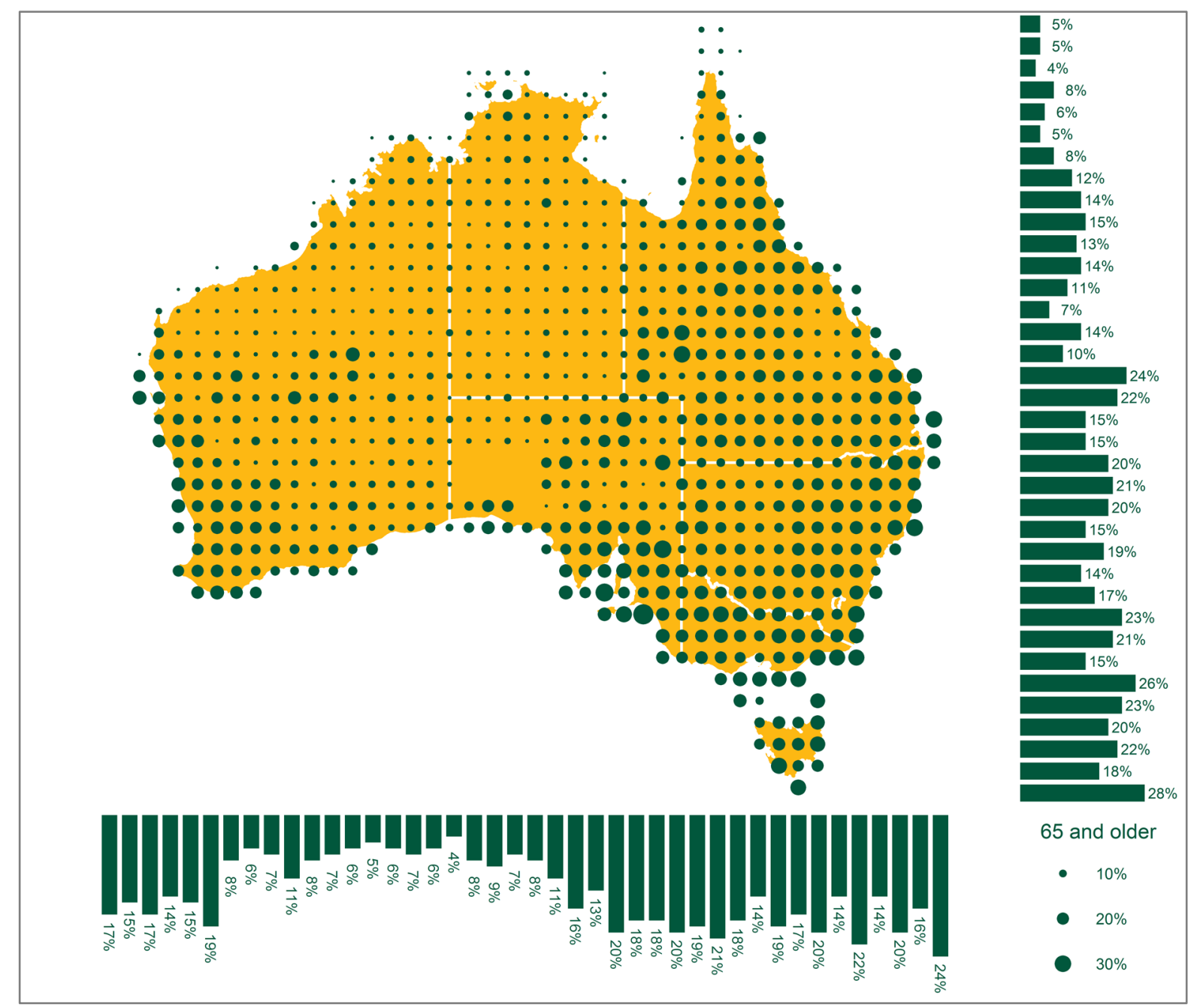

Figure 1: A grid map and marginal histograms of the proportion of Australia's population aged 65 and older Source: Calculated by the author using data extracted from the 2016 census using data from the Australian Bureau of Statistics Census DataPacks (ABS 2016) 


\section{Supplementary Material}

The bespoke script developed to read and prepare the ABS data and Figure 1 is available from the author's online repository at https://rpubs.com/AnthonyKimpton/.

\section{Acknowledgements}

This DemoGraphic is developed through a project funded by the Australian Research Council Linkage Project grant LP160100031 with additional support from the Queensland Department of Transport and Main Roads as industry partner. The analysis and interpretations are solely those of the author and do not necessarily reflect the views and opinions of the Department or any of its employees.

\section{References}

Australian Bureau of Statistics (2016) Census DataPacks. https://datapacks.censusdata.abs.gov.au/datapacks/. Accessed on 15 April 2020.

Australian Institute of Health and Welfare (2018) Older Australia at a glance. https://www.aihw.gov.au/reports/older-people/older-australia-at-a-glance. Accessed on 15 April 2020.

Houghton K and Vonthethoff B (2017) Ageing and work in regional Australia: pathways for accelerating economic growth. http://www.regionalaustralia.org.au/home/ageing-work-regional-australia/. Accessed on 15 April 2020.

Hugo G (2003) Australia's ageing population: some challenges for planners. Australian Planner 40(2): 109118.

Li S, Juhász-Horváth L, Harrison P A, Pintér L and Rounsevell M D A (2016) Population and age structure in Hungary: a residential preference and age dependency approach to disaggregate census data. Journal of Maps 12(sup1): 560-569.

Lambrechts M (2019) How to make a grid map with histograms in R with ggplot. Flowing- Data. https://flowingdata.com/2019/12/16/grid-map-histogram-ggplot/. Accessed on 15 April 2020.

Parliamentary Budget Office (2019) Australia's Ageing Population: Understanding the Fiscal Impacts Over the Next Decade. Report No. 02/2019. Canberra: Parliamentary Budget Office.

Silva B, Javier Gallego F, and Lavalle C (2013) A high-resolution population grid map for Europe. Journal of Maps 9(1): 16-28. 